\title{
PULMONARY ATRESIA, INTACT VENTRICULAR SEPTUM, AND MAJOR AORTOPULMONARY COLLATERALS: MORPHOGENETIC AND SURGICAL IMPLICATIONS
}

\author{
Giovanni B. Luciani, MD, ${ }^{\mathrm{a}}$ Sylvia Swilley, MD, ${ }^{\mathrm{b}}$ and Vaughn A. Starnes, MD, ${ }^{\mathrm{a}}$ Los Angeles and Bellflower, Calif.
}

Pulmonary atresia with intact ventricular septum (PA/ IVS) and pulmonary atresia with ventricular septal defect (PA/VSD) have long been considered two different disease entities. ${ }^{1,2}$ We describe a rare "transitional" case of an infant with pulmonary atresia, intact ventricular septum, and absent ductus arteriosus in whom the pulmonary circulation was supplied by multiple aortopulmonary collaterals (MAPCA). The morphogenetic and surgical aspects of the case are discussed.

A $3.9 \mathrm{~kg}$ full-term male child had cyanosis at birth. Two-dimensional echocardiography showed PA/IVS and hypoplastic right ventricle ( $\mathrm{RV}$ ) for which the baby had a right modified Blalock-Taussig shunt $(4 \mathrm{~mm}$ polytetrafluoroethylene). Failure to thrive and cardiomegaly on chest $\mathrm{x}$-ray film, with oxygen saturations ranging from $82 \%$ to $88 \%$, persisted despite digitalis and furosemide therapy. Cardiac catheterization at 3 months showed a hypoplastic RV with multiple coronary sinusoids (Fig. 1) and hypoplastic and discontinuous central pulmonary arteries with two major MAPCA separately supplying the right and left lung (Fig. 2). Systemic RV pressures (90/4 to $14 \mathrm{~mm} \mathrm{Hg}$ ) were measured. At 4 months, the patient underwent bilateral unifocalization of the pulmonary blood supply and reconstruction of the discontinuous intrapericardial pulmonary arteries, as a stage toward a univentricular palliation through a transverse bilateral thoracosternotomy. Because of persisting heart failure, repeat catheterization was done, which showed successful bilateral unifocalization, with markedly elevated pulmonary arterial pressures (mean right pulmonary artery $35 \mathrm{~mm} \mathrm{Hg}$, mean left pulmonary artery $26 \mathrm{~mm} \mathrm{Hg}$ ) and abnormal arborization of the pulmonary vasculature. Given the clinical and hemodynamic findings, the patient was considered a poor candidate for a cavopulmonary connection, and at time this article was written he continued to have congestive failure and was awaiting heart-lung transplantation.

Although PA/IVS has been invariably associated with confluent pulmonary arteries and a patent ductus arteriosus, PA/VSD has been typically described with varying degrees of underdevelopment of the pulmonary arteries going from mildly hypoplastic but confluent vessels supplied by a patent ductus arteriosus to MAPCA with severely hypoplastic, nonconfluent, or even absent pulmonary arteries. ${ }^{1,2}$ These observations have led to the formulation of two different embryogenetic theories. ${ }^{2}$ Ac-

From the Division of Cardiothoracic Surgery, Childrens Hospital, Los Angeles, University of Southern California School of Medicine, Los Angeles, ${ }^{a}$ and the Division of Pediatric Cardiology, Kaiser Permanente, Bellflower, ${ }^{\mathrm{b}}$ Calif.

J THORAC CARDIOVASC Surg 1995;110:853-4

Copyright (C) 1995 by Mosby-Year Book, Inc.

$0022-5223 / 95 \$ 5.00+0 \quad \mathbf{1 2 / 5 4 / 6 5 1 8 8}$

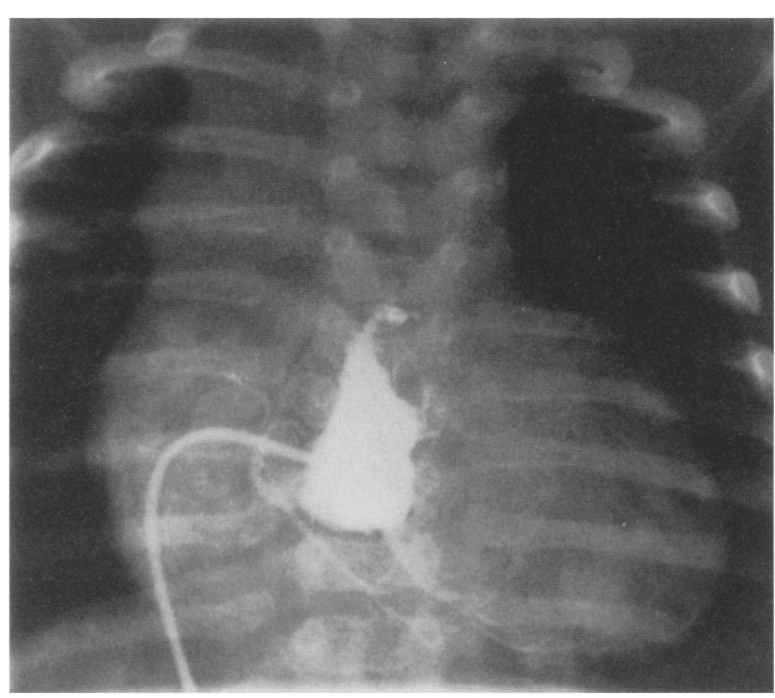

Fig. 1. Right ventriculogram in posteroanterior projection showing marked right ventricular hypoplasia, intact ventricular septum, and sinusoids connecting to coronary circulation.

cordingly, PANSD, an extreme form of tetralogy of Fallot, is due to unequal partitioning of the distal bulbus cordis and truncus arteriosus leading to obliteration of the right ventricular outflow tract (RVOT) with involution of the sixth aortic arches and maintenance of the primitive connections of the pulmonary plexus to the descending thoracic aorta through the intersegmental arteries. In PA/IVS, the nearly normal aspect of the pulmonary arteries, the often normal pulmonary valve, and the normal PDA with absence of MAPCA suggest a different disease entity and a later gestational development. ${ }^{2}$ This case challenges previously proposed embryogenetic theories. We speculate that either PA/IVS and PA/VSD are not two different disease entities but just two extremes of a spectrum in which "transitional" forms, like the present one, can occur, or, more likely, membranous PA/IVS and muscular PA/IVS with diminutive RV and coronary sinusoids are two different disease entities. In particular, the muscular forms of PA/IVS may represent a true developmental error that occurs early during gestational life and in which the patterns of pulmonary blood supply may resemble those found in PA/VSD. Support to the latter theory is lended by the absence of a true pulmonary valve, the presence of coronary sinusoids implying long-standing fetal intraventricular hypertension, and the presence of a long and sigmoid-shaped ductus arteriosus, suggesting 

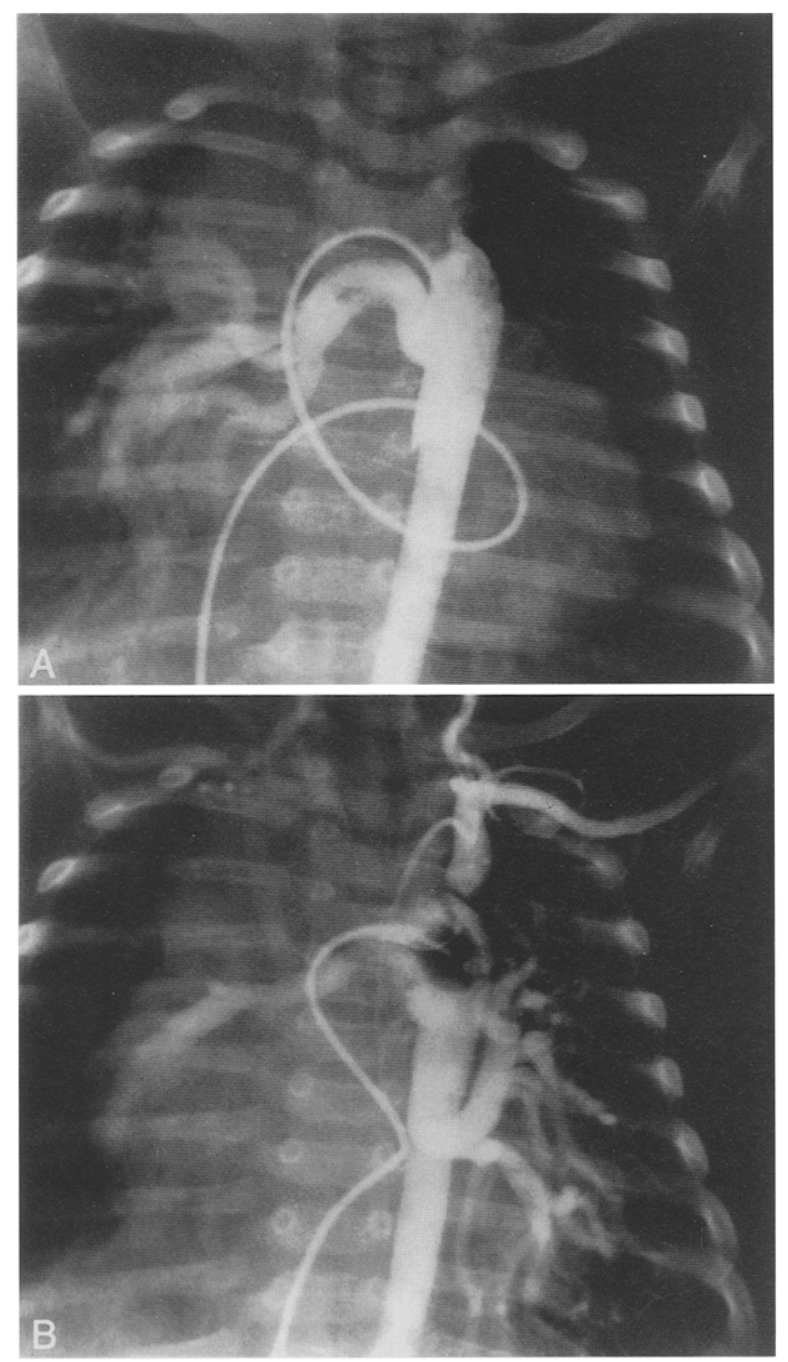

Fig. 2. Aortogram in posteroanterior projection showing patency of the right modified Blalock-Taussig shunt and two large aortopulmonary collateral arteries distributing to the right (A) and left (B) lung.

retrograde flow to the pulmonary arteries, or its absence with MAPCA supplying the lungs. All these conditions are described in muscular forms of PA/IVS.

The current surgical management of PA/IVS includes palliation with a systemic-pulmonary artery shunt or a RVOT procedure. ${ }^{3,4}$ Because of the diminutive RV cavity size, although a shunt placement was necessary in the present case, the ability of a concomitant RVOT procedure to affect RV growth and ultimately allow biventricular repair was questioned. ${ }^{3,4}$ Although the degree of $\mathrm{RV}$ hypoplasia at angiography precluded a future anatomic repair, the hypoplasia of the pulmonary arteries and the presence of MAPCA warranted further palliative surgery, both to allow for appropriate growth of the pulmonary vasculature and to control the excessive volume load on the left ventricle. As is similar with PA/VSD with MAPCA, bilateral unifocalization with reconstruction of the intrapericardial pulmonary arteries was used to recruit the entire pulmonary vascular bed and afford it a chance to grow. ${ }^{4}$ Unlike PA/VSD/MAPCA, in which the RV is rarely small and never diminutive, the goal of the palliation was to improve the pulmonary circulation so as to eventually offer the patient a bidirectional or total cavopulmonary connection. ${ }^{3}$ The proportion of patients with PA/VSD/MAPCA who do not become suitable for anatomic repair because of abnormality in pulmonary arterial branching patterns ranges between $30 \%$ and $50 \%$ in the different series. ${ }^{4}$ Accordingly, because of the persistence of heart failure and abnormal arborization of the intraparenchymal pulmonary arteries after complete unifocalization, the patient was listed for heart-lung transplantation.

This rare case of PA/IVS with MAPCA suggests the existence of alternative embryogenetic mechanisms for different forms of PA/IVS. Staged surgical management of this lesion aiming at orthoterminal palliation ultimately depends on the peripheral arborization of the pulmonary arteries.

\section{REFERENCES}

1. Van Praagh R, Ando M, Van Praagh S, et al. Pulmonary atresia: anatomic considerations. In: Kidd BSL, Rowe RD, eds. The child with congenital heart disease after surgery. New York: Futura, 1976:103-34.

2. Kutsche LM, Van Mierop LHS. Pulmonary atresia with and without ventricular septal defect: a different etiology and pathogenesis for the atresia in the 2 types? Am J Cardiol 1983;51:932-5.

3. Hanley FL, Sade RM, Blackstone EH, Kirklin JW, Freedom RM, Nanda NC. Outcomes in neonatal pulmonary atresia with intact ventricular septum: a multiinstitutional study. J THORAC CARDIOvaSC Surg 1993; 105:406-27.

4. Puga FJ, Leoni FE, Julsrud PR, Mair DD. Complete repair of pulmonary atresia, ventricular septal defect, and severe peripheral arborization abnormalities of the central pulmonary arteries: experience with preliminary unifocalization procedures in 38 patients. J THORAC Cardiovasc Surg 1989;98:1018-29. 